\title{
Green manufacturing: uma análise da produção científica e de tendências para o futuro
}

\author{
Diogo Aparecido Lopes Silva ${ }^{a^{*}}$, Eraldo Jannone da Silva ${ }^{b}$, Aldo Roberto Ometto ${ }^{c}$ \\ a*diogo.apls@hotmail.com, USP, Brasil \\ beraldojs@sc.usp.br, USP, Brasil \\ caometto@sc.usp.br, USP, Brasil
}

\section{Resumo}

0 objetivo deste artigo foi realizar uma análise da produção científica internacional na área de green manufacturing. Foi utilizada a base de dados Web of Science, tendo sido mapeados 239 artigos no período de 1991 a 2013. As publicações envolveram 589 autores, de 36 países, 246 instituições e 142 diferentes periódicos/conferências. Destaca-se que 51,4\% dos artigos foram publicados nos últimos cinco anos. Entre os países mais prolíficos destacaram-se os Estados Unidos e a China. 0 periódico mais prolífico foi o Journal of Cleaner Production, com 19 artigos no período. Sobre a análise de autoria, destacou-se a categoria one-timers, com 85,7\% das publicações. A maior produção foi de 11 artigos por autor e o mais citado recebeu 368 citações. Conclui-se que a temática teve uma ascensão expressiva nos últimos anos, com foco em estudos teóricos e teórico-empíricos.

Palavras-chave

Manufatura sustentável. Manufatura ambientalmente sustentável. Bibliometria. Revisão de literatura.

\section{Introdução}

A manufatura ambientalmente sustentável é um tópico de pesquisa usualmente referenciado na literatura pelos termos em inglês green manufacturing e sustainable manufacturing (Dornfeld, 2012; International Trade Administration, 2010). Seu surgimento se deve à necessidade de integrar questões de sustentabilidade ambiental nos processos produtivos de fabricação.

Merchant et al. (2005) relatam sobre a evolução histórica do tema manufatura salientando o enfoque mais voltado à eficiência operacional dos processos produtivos com base em métricas como a redução do tempo de produção e a minimização de custos operacionais. Já a preocupação com a sustentabilidade dos processos produtivos passou a ter maior destaque somente a partir da década de 1990.

0 conceito manufatura sustentável atualmente é visto como a manufatura de produtos que utilizam processos produtivos menos impactantes ambientalmente, socialmente e que se mostram economicamente viáveis (International Trade Administration, 2010). No âmbito da sustentabilidade ambiental, destacam-se as iniciativas que promovem a conservação de recursos naturais e de energia (Dornfeld, 2012).

Apesar de sua origem nos anos de 1990, as pesquisas voltadas à manufatura ambientalmente sustentável têm se intensificado apenas nos últimos 5 anos (conforme detalhamento na seção 4.1). Assim, torna-se importante investigar em que estado se encontra a produção científica sobre green manufacturing (GM).

A questão de pesquisa que guiou este trabalho foi: Como a produção científica em GM tem se configurado? Como objetivo geral, este artigo visa analisar a produção científica atual e as tendências futuras no tema de estudo.

\section{Green manufacturing}

A manufatura sustentável integra os conceitos de manufatura e sustentabilidade. Manufatura é definida por Kalpakjian (2001, p. 2) como: “[...] o uso de máquinas, ferramentas e mão de obra para 
a produção de produtos para uso ou venda [...]", referindo-se normalmente à produção em escala industrial, onde recursos materiais e energéticos são transformados em produtos acabados em larga escala. Sustentabilidade foi inicialmente definida pela Comissão de Brundtland como

[...] o atendimento às necessidades presentes sem que essas comprometam a possibilidade de as gerações futuras satisfazerem as suas próprias necessidades. (World Commission on Environment and Development, 1987, p. 37).

A sustentabilidade é apresentada como um conceito sistêmico que visa o balanceamento entre os aspectos ambientais, econômicos e sociais, conhecidos pela expressão Triple Botton Line.

$A$ indústria de manufatura envolve diversos setores da economia, a Sustainable Manufacturing Initiative (2011) classifica-os em 15 subdivisões, como a produção de alimentos, de máquinas e equipamentos, de produtos químicos, de produtos metálicos etc. Sobre sua representatividade socioeconômica, é responsável por empregar mundialmente 650 milhões de pessoas (United Nations Environment Programme, 2011), e na Europa, representa 22\% do produto interno bruto e $70 \%$ dos empregos diretos e indiretos (Manufuture, 2004).

Numa revisão sobre a evolução histórica da indústria de manufatura nos últimos 60 anos, Merchant et al. (2005) concluíram sobre a importância do uso da tecnologia nos processos produtivos, exaltando a automação industrial, pois facilita o gerenciamento e o monitoramento dos processos produtivos em tempo real de fabricação. Também citam a importância da introdução do conceito lean manufacturing, que permitiu a redução de tempos improdutivos e do desperdício no consumo de recursos. Porém, a evolução ocorreu com foco em métricas tradicionais como custo e qualidade, não prevendo a demanda por novas métricas, por exemplo, baseadas na integração de aspectos de sustentabilidade.

A indústria de manufatura, apesar de sua representatividade socioeconômica, é responsável por relevantes impactos ambientais. Os processos de manufatura destacam-se pelos altos níveis de consumo de energia e pelas emissões de gases de efeito estufa (GEEs). Nos Estados Unidos, 21\% do consumo total de recursos energéticos e $26 \%$ do total de GEEs emitidos pelo país são tributados à indústria de manufatura (Energy Information Administration, 2010). No Brasil, para Araújo \& Oliveira (2012), as atividades de manufatura também geram relevantes impactos ambientais, sendo que entre 1994 e 2007 as emissões de carbono na indústria brasileira cresceram
77\%, passando elas a terem importância muito maior como poluidoras.

Jelinski et al. (1992) foram um dos primeiros a apresentar o termo sustainable manufacturing, todavia, relacionando-o apenas com a dimensão ambiental da sustentabilidade. Os autores apresentaram o termo como parte da ecologia industrial. Contudo, o conceito manufatura sustentável tem evoluído e atualmente integra as dimensões social e econômica, sendo definida como:

\section{[...] a manufatura de produtos utilizando materiais e processos que minimizem os impactos negativos sobre o meio ambiente, promovendo a conservação de energia e dos recursos naturais, a segurança dos trabalhadores, dos consumidores e da comunidade, $\mathrm{e}$ que se mostra economicamente viável. (International Trade Administration, 2010, p. 1).}

Apesar de sua definição mais recente englobar as três dimensões da sustentabilidade, o termo sustainable manufacturing está mais relacionado ao âmbito ambiental. Assim, diversos autores (Bergmiller, 2006; Dornfeld, 2012; Rusinko, 2007) têm empregado mais o termo green manufacturing (GM). Todavia, há outros termos na literatura que se mostram equivalentes a GM, como: sustainable production (Pusavec et al., 2010), environmentally conscious manufacturing (Florida, 1996; Gungor \& Gupta, 1999; llgin \& Gupta, 2010; Sarkis, 1999) e environmentally benign manufacturing (Allen et al., 2002; Gutowski et al., 2005).

A visão sobre $\mathrm{GM}$ tem se modificado ao longo do tempo. Baseado no Sustainable Manufacturing Initiative (2011), a Figura 1 apresenta um diagrama referente à sua evolução.

A partir da Figura 1, verifica-se que a visão sobre o conceito GM tem evoluído conforme o surgimento de novas práticas de gestão ambiental. No início, eram focadas apenas em soluções fim-de-tubo, passando

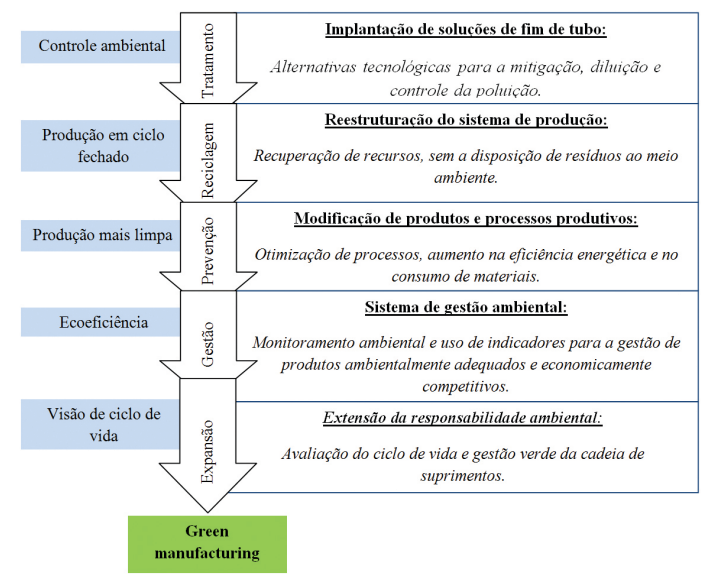

Figura 1. Evolução da visão e das práticas de GM. Fonte: adaptado de Sustainable Manufacturing Initiative (2011). 
pela produção em ciclo fechado, a produção mais limpa $(\mathrm{P}+\mathrm{L})$ e a ecoeficiência industrial, nos dias atuais evoluiu-se com sua integração à visão de ciclo de vida (CV) do produto.

Para diversos autores (Araújo \& Oliveira, 2012; Rusinko, 2007; Silva \& Amaral, 2009; Silva et al., 2013), a GM é alcançada por meio de práticas de produção mais limpa $(\mathrm{P}+\mathrm{L})$, pois a $\mathrm{P}+\mathrm{L}$ tem foco sobre o processo produtivo e permite o aumento da eficiência no uso dos recursos naturais e a minimização na geração de resíduos, possibilitando uma melhor ecoeficiência industrial.

Considerando a metodologia de $\mathrm{P}+\mathrm{L}$ para subsidiar a produção sustentável, Araújo \& Oliveira (2012) desenvolveram um método para a avaliação de desempenho em sustentabilidade de processos de manufatura, com foco na construção de relatórios de sustentabilidade para apoiar o processo de tomada de decisão gerencial. Silva \& Amaral (2009) desenvolveram uma metodologia integrada para a avaliação dos impactos ambientais e dos custos de processos de fabricação a partir da P+L. Silva et al. (2013) propuseram uma metodologia de $\mathrm{P}+\mathrm{L}$ integrando ferramentas da qualidade, no intuito de melhorar sua aplicabilidade na indústria. Outros autores, como Florida (1996) e King \& Lenox (2001), relacionaram a $\mathrm{GM}$ com a P+L e com o lean manufacturing, que foca na redução do desperdício de tempo e de recursos, o que por consequência também pode promover a melhoria ambiental dos processos de fabricação. Contudo, tais estudos não integraram a visão de $\mathrm{CV}$ em seu escopo.
A visão de CV pode ser entendida como o conjunto de todas as etapas necessárias para que um produto cumpra sua função, numa perspectiva tipo cradle-to-grave. A Avaliação do Ciclo de Vida $(A C V)$ é uma técnica que considera o conceito de CV em sua metodologia. A ACV pode ser definida como técnica para a

[...] compilação e avaliação das entradas, das saídas e dos impactos ambientais potenciais de um sistema de produto ao longo de seu ciclo de vida." (Associação Brasileira de Normas Técnicas, 2009, p. 6).

As entradas incluem a mensuração do consumo de materiais e de energia e as saídas, os fluxos de produtos e coprodutos, emissões gasosas, efluentes líquidos, resíduos sólidos, perdas de energia.

A inserção da ACV para subsidiar a GM é recente e tem sido objeto de estudo em diversas publicações (Duflou et al., 2012; Silva et al., 2012; Murray et al., 2012), com foco na redução do consumo de recursos e na minimização da geração de resíduos (Dornfeld, 2012; Murray et al., 2012), e por meio da otimização do CV dos produtos (Linke et al., 2012; Silva et al., 2012), conforme a representação esquemática da Figura 2.

Na Figura 2, a visão sobre GM considera a produção em ciclo fechado, onde nenhum resíduo é disposto no meio ambiente (zero landfill). Portanto, considera-se como fluxo evitado a disposição final de resíduos. Ao mesmo tempo, a obtenção de recursos na fase de pré-produção é considerada como fluxo minimizado, devido à redução das atividades de extração e transformação de recursos naturais. Isso

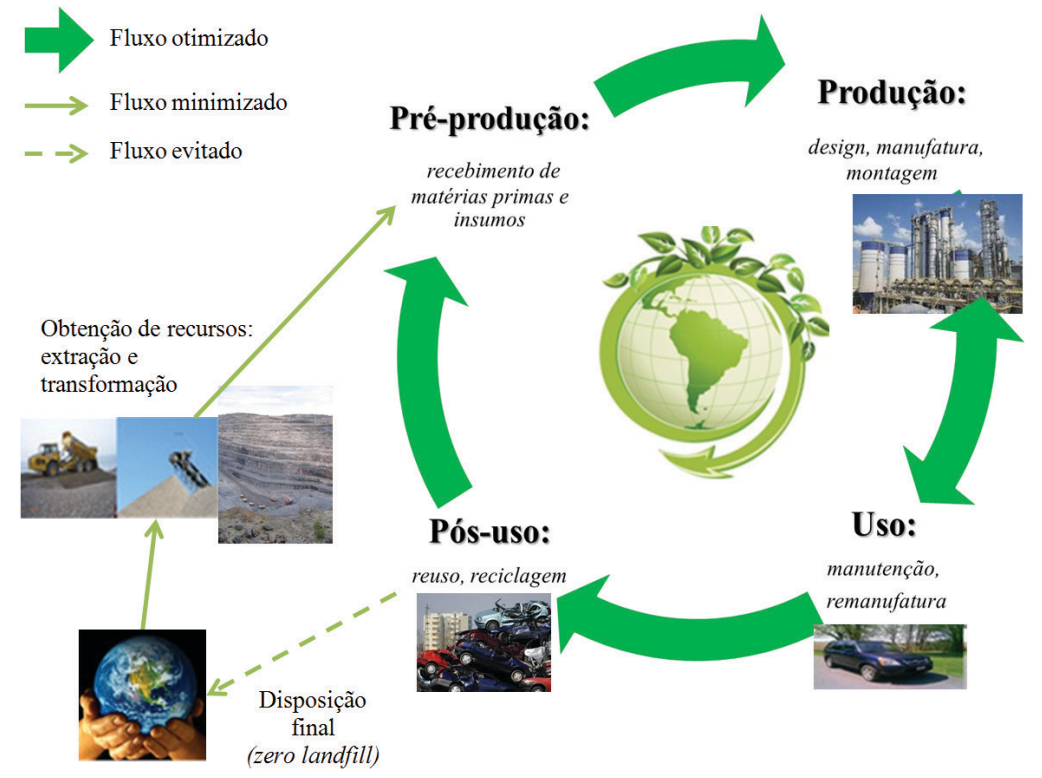

Figura 2. GM e a visão de CV. Fonte: adaptado de National Institute of Standards and Technology - NIST (2013). 
ocorre por, durante o CV do produto, nas etapas de uso e pós-uso, serem consideradas estratégias como a remanufatura, o reuso e a reciclagem de produtos e resíduos, o que reduz a demanda pelo consumo de matérias-primas. Por fim, na etapa de produção, é necessário que os processos de manufatura sejam monitorados e controlados a fim de otimizarem-se os fluxos de materiais e de energia consumidos e as perdas durante a produção, sendo, nesse ponto, importante para Dornfeld (2012) integrar o uso da ACV com outras técnicas/ferramentas.

Apesar da visão mais recente sobre GM integrar o conceito de CV, o esquema da Figura 2 não deve ser assumido como um sistema de produção estabelecido. Bergmiller (2006) cita que diferentemente do Lean Manufacturing, que é baseado no sistema Toyota de produção, a GM não pode ser visto como um sistema de produção, já que historicamente sempre foi encarado como o resultado de práticas de gestão ambiental nos processos de manufatura (conforme a Figura 1), não apresentando um modelo que possa ser generalizado. Para a consolidação de um modelo de GM, é necessária sua integração com ferramentas gerencias adequadas, o que, para Garetti \& Taisch (2012), tem se mostrado uma das principais barreiras e desafios na atualidade. 0 uso isolado de técnicas/ ferramentas de gestão ambiental como a ACV e a $\mathrm{P}+\mathrm{L}$ não permitem a consolidação de um modelo consensual de GM.

Assim, os resultados apresentados neste artigo podem subsidiar pesquisas, por exemplo, com foco na consolidação de modelos de GM. Afinal, uma análise detalhada da produção científica internacional sobre GM é realizada neste artigo, apontando as principais publicações e suas características, e gerando insights que podem contribuir para futuras pesquisas na área.

\section{Material e métodos}

A metodologia adotada para este trabalho baseia-se na pesquisa bibliográfica e na aplicação de procedimentos de bibliometria estruturados por meio das etapas apresentadas na Figura 3. Para a definição da amostra de estudo foi utilizada a ferramenta Web of Knowledge, base de dados Web of Science, para o período de publicações até dezembro de 2013. Depois, para a análise bibliométrica, foi utilizado o software HistCite versão 11.12.07.

$\mathrm{Na}$ etapa 1 foram selecionadas apenas as publicações conforme os critérios: formato texto completo, tipo de documento - artigo, idioma - inglês e tipo de publicação - academic journal ou proceedings paper. As palavras-chave adotadas foram sustainable manufacturing e green manufacturing, utilizadas nos critérios de busca título (title) e assunto (topic). Posteriormente, outras palavras-chave foram inseridas na busca: sustainable production, environmentally conscious manufacturing e environmentally benign manufacturing, sendo a adoção justificada pela similaridade de resultados obtidos em termos de conteúdo e abordagem dos artigos em relação aos obtidos utilizando as palavras-chave principais.

Com base nos resultados da busca, como último critério para a definição da amostra, a partir do resumo de cada artigo foram selecionados apenas os que apresentassem alguma contribuição conforme a visão e as práticas de GM mostradas na Figura 1, seção 2.

Ao todo, foram selecionados 239 artigos, sendo que a partir dos resultados da busca foi realizada a etapa 2 da pesquisa. As categorias de análise para a classificação das publicações foram: data de publicação, autoria da obra, afiliação dos autores, nome do periódico/conferência.

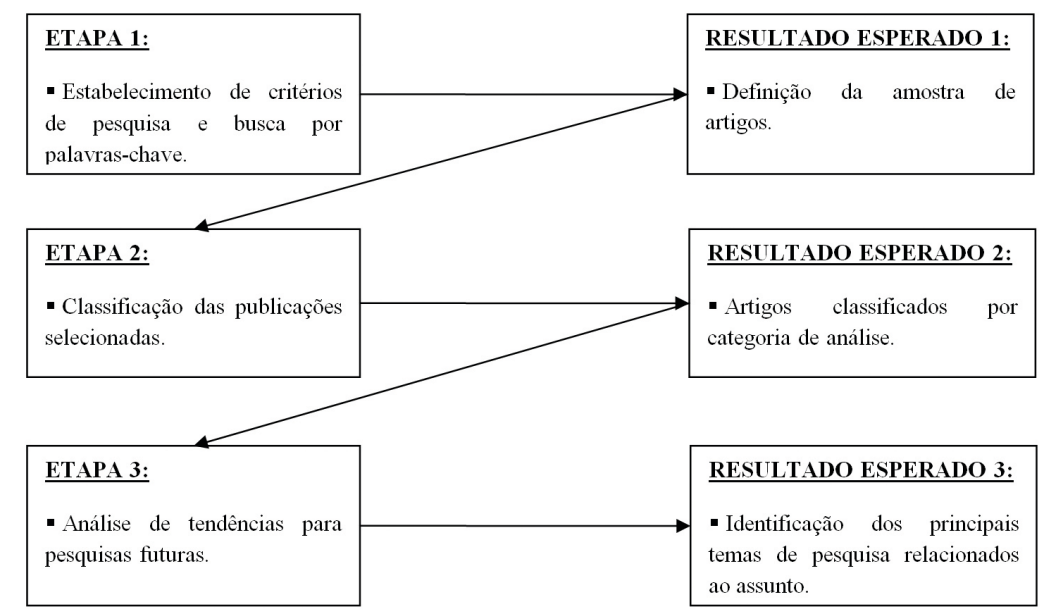

Figura 3. Representação das fases de desenvolvimento da pesquisa. 
Para a categoria autoria da obra classificaram-se os autores conforme a produtividade na área (quantidade de artigos publicados e quantidade de citações recebidas) e segundo o enquadramento produtivo: continuantes, transientes, entrantes, one-timers e retirantes (Walter \& Back, 2013), conforme a Tabela 1. Para a análise do número de citações, foram adotados indicadores bibliométricos do software HistCite destacados na Tabela 2.

$\mathrm{Na}$ etapa 3 foi realizada a parte final do trabalho, focada na identificação de tendências futuras para pesquisas em GM. Para tanto, fez-se o cruzamento dos temas dos principais artigos publicados (com mais LCS) junto aos temas dos artigos mais recentemente publicados.

\section{Resultados e discussões}

\subsection{Análise do evolutivo, país de origem e do tipo de instituição}

A Figura 4 mostra o evolutivo da produção científica sobre GM no período 1991-2013 (22 anos). 0 período $1991-2000$ contabilizou $11,7 \%$ do total de artigos e a quantidade máxima de publicações ocorridas foi de 6 artigos em 1999. Todavia, desde 2001 que ao menos 7 artigos sobre o tema são publicados anualmente e 51,4\% das publicações estão concentradas nos últimos 5 anos, o que indica uma ascensão do tema nos últimos anos.

A Tabela 3 mostra a caracterização da amostra de artigos conforme o país de origem dos estudos.

Foram identificados 36 países e contabilizadas 284 publicações. Conforme a Tabela 3, 74,3\% das publicações se concentram em: Estados Unidos, China, Reino Unido, Japão, Austrália, Canadá, Índia, ltália e Alemanha. Outros 24 artigos estão distribuídas entre países que publicaram de 1 a 3 artigos cada. Além disso, ressalta-se que o Brasil contribuiu com apenas 1 artigo na amostra, caracterizando sua baixa representatividade na área.

A Tabela 4 mostra a caracterização dos artigos estudados conforme o tipo de instituição envolvida.

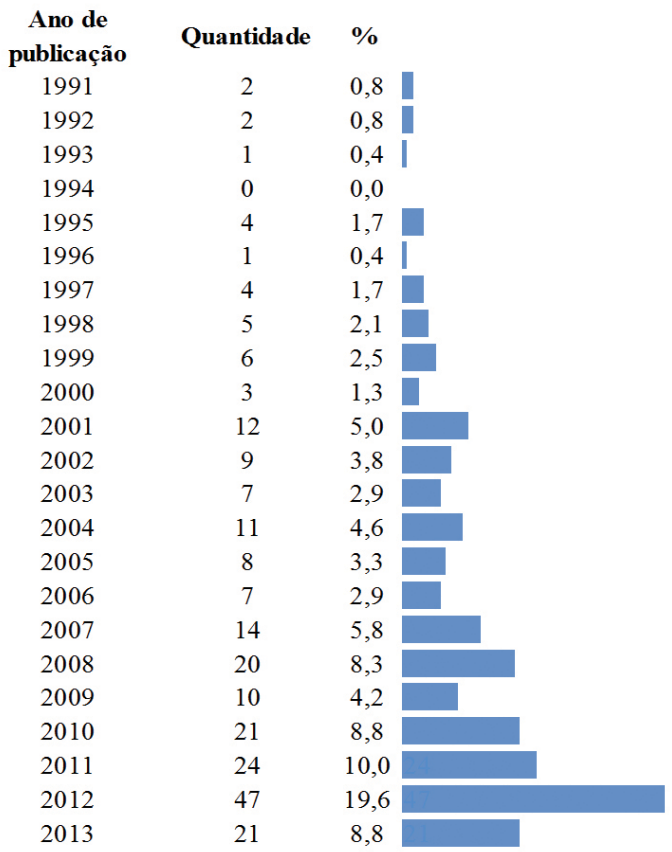

Figura 4. Evolutivo da produção científica em GM.

Tabela 1. Enquadramento produtivo: critérios para a classificação dos autores da área.

\begin{tabular}{|c|c|c|}
\hline Categoria & Definição & Critérios para classificação \\
\hline Entrantes & $\begin{array}{l}\text { Novos pesquisadores na área, com publicação de pelo menos } 2 \text { artigos } \\
\text { nos últimos } 4 \text { anos. }\end{array}$ & $\begin{array}{l}\geq 2 \text { artigos de } 2010 \text { a } 2013 \\
\text { Sem publicações até } 2009\end{array}$ \\
\hline Transientes & $\begin{array}{l}\text { Pesquisadores relativamente permanentes na área, com publicações de } \\
\text { pelo menos } 2 \text { artigos em, no máximo, } 5 \text { anos, tendo publicações tanto } \\
\text { nos últimos } 4 \text { anos quanto antes. }\end{array}$ & $\begin{array}{l}\geq 2 \text { artigos em até } 5 \text { anos diferentes } \\
\geq 1 \text { artigo de } 2010 \text { a } 2013 \\
\geq 1 \text { artigo até } 2009\end{array}$ \\
\hline Continuantes & $\begin{array}{l}\text { Pesquisadores consolidados na área, com publicação de pelo menos } 2 \\
\text { artigos em } 5 \text { ou menos anos diferentes, inclusive nos } 4 \text { últimos anos. }\end{array}$ & $\begin{array}{l}\geq 2 \text { artigos em } \leq 5 \text { anos } \\
\geq 1 \text { artigo de } 2010 \text { a } 2013\end{array}$ \\
\hline One-timers & $\begin{array}{l}\text { Pesquisadores esporádicos, com publicação de apenas } 1 \text { artigo no } \\
\text { período de análise. }\end{array}$ & 1 artigo até 2013 \\
\hline Retirantes & $\begin{array}{l}\text { Pesquisadores que estão deixando a área, com publicações de ao menos } \\
2 \text { artigos, porém, com nenhuma publicação nos últimos } 4 \text { anos. }\end{array}$ & $\begin{array}{l}\geq 2 \text { artigos até } 2009 \\
\text { Sem publicações de } 2010 \text { a } 2013\end{array}$ \\
\hline
\end{tabular}

Tabela 2. Indicadores bibliométricos adotados na análise de citações.

\begin{tabular}{cl} 
Indicador & \multicolumn{1}{c}{ Definição } \\
\hline LCS & $\begin{array}{l}\text { Total de citações locais de um artigo da amostra (Local Citation Scores - LCS). Uma citação local é contabilizada quando } \\
\text { um artigo da amostra apresenta citação de algum outro artigo de dentro da mesma amostra. Esse indicador mede o quão } \\
\text { relevante é o artigo selecionado dentro da amostra estudada. }\end{array}$ \\
GCS & Soma de todas as citações (Global Citation Scores - GCS) de um artigo dentro da base de dados Web of Science. \\
\hline
\end{tabular}


As publicações mostram-se distribuídas entre muitas instituições distintas. Foram identificadas 246 instituições, sendo 161 universidades $(65,4 \%)$, 47 institutos de pesquisa $(19,1 \%)$ e 38 empresas $(15,4 \%)$. Conforme a Tabela 4, 256 publicações $(69,4 \%)$ foram produzidas por universidades, 72 (19,5\%), por institutos de pesquisa e $41(11,1 \%)$, por empresas.

Entre as universidades, os destaques foram a University of California (Estados Unidos), University of Chongqing (China), Wuhan University of Science and Technology (China), Technical University of Denmark (Diamarca) e University of Cranfield (Reino Unido), totalizando de 7 a 13 publicações cada. As demais 156 universidades somaram de 1 a 5 artigos cada. Sobre a University of California situada em Berkely, destaca-se o Laboratory for Manufacturing and

Tabela 3. Caracterização dos artigos: país de origem.

\begin{tabular}{lcc}
\multicolumn{1}{c}{ País } & No de publicações & $\%$ \\
\hline Estados Unidos & 81 & 28,5 \\
China & 51 & 18,0 \\
Reino Unido & 18 & 6,3 \\
Japão & 12 & 4,2 \\
Austrália & 10 & 3,5 \\
Canadá & 10 & 3,5 \\
India & 10 & 3,5 \\
ltália & 10 & 3,5 \\
Alemanha & 9 & 3,2 \\
Dinamarca & 7 & 2,5 \\
Bélgica & 5 & 1,8 \\
Suécia & 5 & 1,8 \\
França & 4 & 1,4 \\
Holanda & 4 & 1,4 \\
Coreia do Sul & 4 & 1,4 \\
Espanha & 4 & 1,4 \\
Suíça & 4 & 1,4 \\
Taiwan & 4 & 1,4 \\
Outros & 24 & 8,5 \\
\hline
\end{tabular}

Sustainability (LMAS), responsável pela maioria das publicações. A University of Chongping, localizada na província de Chongping, destaca-se pelas pesquisas do Institute of Manufacturing Engineering e pelo State Key Laboratory of Mechanical Transmission. A Wuhan University of Science and Technology (WUST), situada em Wuhan, destaca-se por conta das publicações realizadas pelo Manufacturing Engineering Institute. A Technical University of Denmark, localizada em Lyngby, destaca-se por conta das publicações do Department of Manufacturing Engineering and Management. Por fim, a University of Cranfield, situada em Cranfield, destaca-se principalmente pelas publicações do Manufacturing and Materials Department.

Entre os institutos de pesquisa, os destaques foram para o National Institute of Standards and Technology - NIST (Estados Unidos) e o National Institute of Technology, Tiruchirappalli - NITT (Índia), os quais produziram 4 artigos cada. Os demais 45 institutos de pesquisa contribuíram com de 1 a 3 artigos cada. 0 NIST, situado em Gaithersburg, realiza pesquisa em diversas áreas, incluindo o campo tecnologia e meio ambiente para processos de manufatura. Já o NITT, localizado em Tiruchirappalli, destaca-se pelas publicações realizadas pelo Department of Production Engineering.

Sobre a produção científica vinculada a empresas, os destaques foram: DSM Innovative Synthesis B. V. (Holanda), GlaxoSmithKline Inc. (Estados Unidos) e SKF Sverige AB (Suécia), que contribuíram com 2 publicações cada. As demais 35 empresas produziram apenas 1 artigo cada. A DSM Innovative Synthesis B. V., situada na cidade de Geleen, é uma indústria de produtos químicos com foco no setor farmacêutico. A GlaxoSmithKline Inc. é uma empresa situada em diversos países, todavia, merecem destaque as sedes nos Estados Unidos - Filadélfia e Carolina do

Tabela 4. Caracterização dos artigos: instituições envolvidas.

\begin{tabular}{cllcc}
\hline \multicolumn{1}{c}{ Tipo de instituição } & \multicolumn{1}{c}{ Nome } & \multicolumn{1}{c}{ Pais } & No de publicações & $\%$ \\
\hline Universidades & University of California & Estados Unidos & 13 & 5,4 \\
& University of Chongqing & China & 10 & 34,9 \\
& Wuhan University of Science and Technology & China & 9 & 3,5 \\
& Technical University of Denmark & Dinamarca & 7 & 2,7 \\
& University of Cranfield & Reino Unido & 7 & 2,7 \\
& Outros & Múltiplos & 210 & 82,0 \\
Institutos de pesquisa & National Institute of Standards and Technology & Estados Unidos & 256 & 100 \\
& National Institute of Technology, Tiruchirappalli & India & 4 & 5,5 \\
& Outros & Múltiplos & 4 & 5,5 \\
Empresas & & TOTAL & 64 & 89,0 \\
& DSM Innovative Synthesis B. V. & Holanda & 72 & 100 \\
& GlaxoSmithKline Inc. & Estados Unidos & 2 & 4,9 \\
& SKF Sverige AB & Suécia & 2 & 4,9 \\
& Outros & Múltiplos & 2 & 4,9 \\
& & TOTAL & 35 & 85,3 \\
\hline
\end{tabular}


Norte -, que apresentaram publicações sobre GM na indústria farmacêutica A empresa sueca SKF Sverige $A B$, situada em Gothenburg, é uma empresa que atua na indústria metal-mecânica, com destaque para o SKF Group Manufacturing Development, responsável pelas publicações mapeadas.

Portanto, o tema GM tem despertado, em escala global, mais interesse em universidades dos Estados Unidos, China, Dinamarca e Reino Unido; em institutos de pesquisa dos Estados Unidos e da Índia; e em empresas da Holanda, Estados Unidos e Suécia. Os Estados Unidos se mostrou o principal atuante nos três tipos de instituições avaliadas.

\subsection{Análise dos periódicos/conferências envolvidos nas publicações}

A Tabela 5 apresenta uma análise das publicações por periódico/conferência, identificando os mais prolíficos.

No total foram identificados 142 periódicos/ conferências, sendo $90(63,4 \%)$ do tipo periódico e $52(36,6 \%)$ do tipo conferência. Desses, os mais prolíficos responderam por 30,8\% do total das publicações, contabilizando de 4 a 19 artigos cada, como mostra a Tabela 5. As principais revistas identificadas foram o Journal of Cleaner Production e o International Journal of Production Research, ambos classificados como periódicos qualis A2 na área de Engenharia 111 (atualização de 2012). As publicações em conferências variaram de 1-3 artigos por evento, não sendo possível identificar um evento específico de destaque. Todavia, como evento científico de referência em GM ressalta-se o CIRP International Conference in Life Cycle Engineering (CIRP LCE), que ocorre a cada dois anos, sendo que em 2013 realizou sua $20^{a}$ edição. 0 evento é mundialmente reconhecido e organizado por instituições de pesquisa vinculadas ao Collège International pour la Recherche en Productique (CIRP - Academia Internacional de Engenharia de Produção), sendo que os anais de cada edição são disponibilizados na base de dados da Springer. Os anais do CIRP LCE não foram considerados na amostra de estudo, pois não estão disponíveis na base de dados Web of Science.

A Figura 5 apresenta um evolutivo das publicações sobre GM para os principais periódicos identificados.

A partir da Figura 5 é possivel notar que os periódicos analisados apresentam nenhum ou no máximo 2 artigos publicados durante toda a década de 1990. Também verifica-se que o maior índice de

Tabela 5. Periódicos/conferências mais prolíficos.

\begin{tabular}{|c|c|c|}
\hline Nome & No de publicações & $\%$ \\
\hline Journal of Cleaner Production (JCP) & 19 & 7,9 \\
\hline International Journal of Production Research (IJPR) & 10 & 4,2 \\
\hline CIRP Annals - Manufacturing Technology (CA - MT) & 7 & 2,9 \\
\hline International Journal of Production Economics (IJPE) & 7 & 2,9 \\
\hline Proceedings of the Institution of Mechanical Engineers, Part B: Journal of Engineering Manufacture (PIME - JEM) & 7 & 2,9 \\
\hline International Journal of Advanced Manufacturing Technology (IJAMC) & 6 & 2,5 \\
\hline International Journal of Life Cycle Assessment (IJLCA) & 5 & 2,1 \\
\hline International Journal of Precision Engineering and Manufacturing (IJPEM) & 5 & 2,1 \\
\hline Manufacturing Engineering (ME) & 4 & 1,7 \\
\hline Production Planning \& Control (PP \& C) & 4 & 1,7 \\
\hline Outros & 166 & 69,2 \\
\hline
\end{tabular}

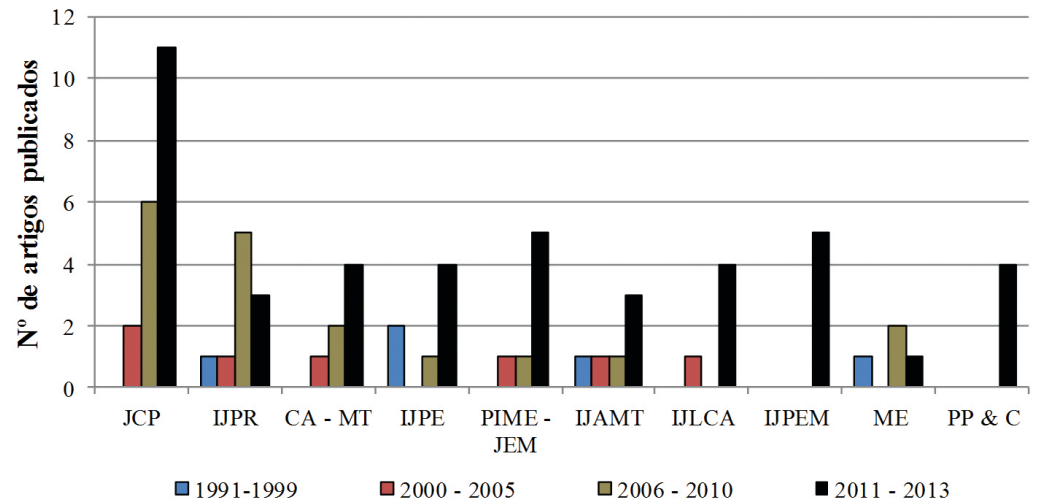

Figura 5. Principais periódicos: evolutivo da produção científica. 
produção foi de 11 artigos, publicados pela revista JCP no período de 2011-2013. Entre 2011-2013 também houve a maior quantidade de artigos publicados, com exceção dos periódicos IJPR e $M E$, o que confirma a maior relevância e ascensão do tema GM principalmente nos últimos anos.

\subsection{Análise de autoria das publicações}

Sobre a análise do enquadramento produtivo, a Tabela 6 apresenta a quantidade de autores identificados por categoria de autoria.

Ao todo foram identificados 589 diferentes autores, sendo observado que os autores entrantes, continuantes, transientes e retirantes apresentam-se pouco expressivos nas publicações mapeadas, diferentemente da categoria one-timers, a qual foi o grande destaque, contabilizando $85,7 \%$ das publicações. Tais constatações contemplam novamente o fato de que o tema GM apenas recentemente foi incorporado à produção científica da academia internacional.

Sobre a produtividade dos autores na área, a Tabela 7 classifica os principais autores conforme a quantidade de artigos publicados.

Verifica-se que os principais autores, conforme a Tabela 7, somam apenas 7 entre os 589 autores mapeados, sendo 2 dos Estados Unidos e cinco da China, que eles respondem por $24,3 \%$ do total de publicações e que o número máximo de artigos publicados por autor variou de 6 a 11. Desse modo, verifica-se que a maior parte $(75,7 \%)$ das publicações está dispersa entre os demais 582 autores $(98,8 \%)$, os quais publicaram de 1 a 4 artigos cada. lsso mostra

Tabela 6. Enquadramento produtivo dos autores na área.

\begin{tabular}{lcc}
\multicolumn{1}{c}{ Categorias } & No de autores & $\%$ \\
\hline Autores entrantes & 20 & 3,4 \\
Autores continuantes & 13 & 2,2 \\
Autores transientes & 13 & 2,2 \\
Autores one-timers & 505 & 85,7 \\
Autores retirantes & 38 & 6,5 \\
Total & 589 & 100 \\
\hline
\end{tabular}

a existência de um grande número de pesquisadores, fato atribuído à grande quantidade de instituições envolvidas nas pesquisas sobre o tema (seção 4.1). Corroborando, acrescenta-se o fato de a maior parte dos autores mapeados terem sido classificados como one-timers, pesquisadores esporádicos e não consolidados na área. Por outro lado, os 7 principais autores da Tabela 7 são autores continuantes.

Considerando-se que a produtividade de um autor deve levar em conta também o número de citações que suas publicações recebem, a Tabela 8 classifica os autores mapeados conforme a quantidade de citações tipo GCS.

Do total das 2.207 citações tipo GCS, a partir da Tabela 8 verifica-se que 19 autores são os principais, com número de citações de 61 a 368. Do total, 13 autores são dos Estados Unidos, 2 do Canadá, 2 da Índia, 1 da Turquia e 1 da Dinamarca, representando juntos por 73,9\% das citações. Desse modo, verifica-se que a maior parte das citações está concentrada numa pequena quantidade de pesquisadores $(3,2 \%$ dos 589 autores mapeados).

Ao analisar a produtividade dos principais autores da área de GM, verificam-se diferentes constatações, conforme o critério de comparação. A partir das Tabelas 7 e 8 observa-se que os autores com maior número de publicações não são os que apresentaram maior número de citações. Na Tabela 8, mais de 90\% dos autores foram classificados como one-timers ou retirantes, sendo que a maior parte de seus artigos datam do período 1990-2007; além disso, apenas 1 autor foi classificado como continuante (Jon Sutherland). De modo distinto, na Tabela 7 todos os autores de destaque foram classificados como continuantes.

Assim, pode-se inferir que os autores da Tabela 7 são os principais pesquisadores da área na atualidade. Já os autores da Tabela 8 estão, em sua maioria, deixando de atuar na área (retirantes) ou não são grandes atuantes (one-timers), porém suas obras, ainda que retrospectivas, servem de base para a continuidade e o desenvolvimento de mais publicações em GM.

Tabela 7. Principais autores na área: número de artigos publicados.

\begin{tabular}{lccc}
\hline \multicolumn{1}{c}{ Autor } & País & No de artigos publicados & $\%$ \\
\hline Feng Liu & China & 11 & 4,6 \\
David Dornfeld & Estados Unidos & 10 & 4,2 \\
Hua Zhang & China & 9 & 3,8 \\
Jon Sutherland & Estados Unidos & 9 & 3,8 \\
Zhigan Jiang & China & 7 & 2,9 \\
Congbo Li & China & 6 & 2,5 \\
Xian-Chun Tan & China & 6 & 2,5 \\
Outros & Múltiplos & 181 & 75,7 \\
\hline
\end{tabular}




\subsection{Análise de tendências para pesquisas futuras}

Esta seção identifica tendências futuras para pesquisas sobre GM. Para tanto, inicialmente foram mapeados os principais temas estudados na área através da seleção dos artigos com maiores valores de LCS (citações locais dentro da amostra pesquisada), conforme mostram a Figura 6 e a Tabela 9. A Figura 6 apresenta o mapa de relacionamento entre as publicações analisadas, considerando os valores de LCS de cada artigo, sendo os principais trabalhos destacados entre círculos.

Na Figura 6, cada artigo foi citado de 0 a 12 vezes, portanto, apresentam valores de LCS de 0 a 12 . Observam-se 9 trabalhos em destaque, os quais em sua maioria foram publicados antes de 2003, apresentando valores de LCS de 6 a 12, conforme detalhes na Tabela 9.

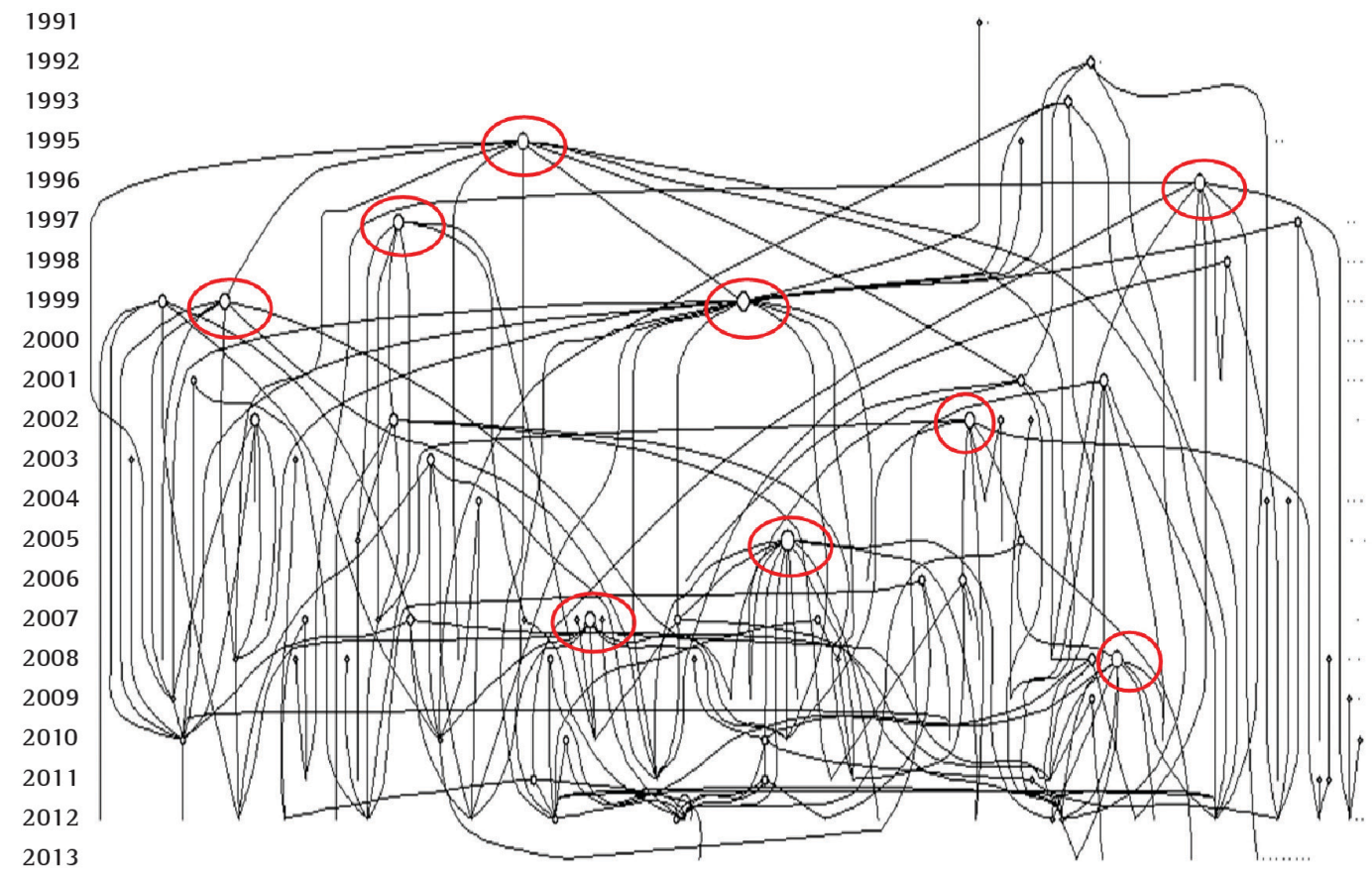

Figura 6. Mapa de relacionamento entre as publicações avaliadas.

Tabela 8. Principais autores na área: número de citações globais recebidas.

\begin{tabular}{|c|c|c|c|}
\hline Autores & Pais & No de citações GCS recebidas & $\%$ \\
\hline Surendra Gupta & Estados Unidos & 368 & 21,7 \\
\hline Askiner Gungor & Estados Unidos & 295 & 17,4 \\
\hline Joseph Sarkis & Estados Unidos & 167 & 9,9 \\
\hline Richard Florida & Estados Unidos & 157 & 9,2 \\
\hline $\begin{array}{l}\text { Robert Klassen } \\
\text { Stephan Vachon }\end{array}$ & Canadá & 95 & 5,6 \\
\hline $\begin{array}{l}\text { G. Dangayach } \\
\text { S. Deshmukh }\end{array}$ & Índia & 86 & 5,1 \\
\hline Mehme Ali llgin & Turquia & 73 & 4,3 \\
\hline Jon Sutherland & Estados Unidos & 73 & 4,3 \\
\hline Bert Bras & Estados Unidos & 65 & 3,8 \\
\hline $\begin{array}{l}\text { Thomas Graedel } \\
\text { L. Jelinski } \\
\text { R. Laudise } \\
\text { D. Mccall } \\
\text { C. Patel }\end{array}$ & Estados Unidos & 65 & 3,8 \\
\hline Timothy Gutowski & Estados Unidos & 64 & 3,7 \\
\hline Paul Sheng & Estados Unidos & 63 & 3,7 \\
\hline Leo Alting & Dinamarca & 61 & 3,6 \\
\hline Outros & Múltiplos & 575 & 7,6 \\
\hline
\end{tabular}


Tabela 9. Principais publicações mapeadas.

\begin{tabular}{|c|c|c|c|c|c|}
\hline & Autoria & Ano & Temas estudados & Tipo de estudo & LCS \\
\hline (1) & Joseph Sarkis & 1995 & $\begin{array}{l}\text { Manufacturing strategy and environmental } \\
\text { consciousness. }\end{array}$ & Teórico & 9 \\
\hline (2) & Richard Florida & 1996 & $\begin{array}{l}\text { Lean and green: the move to environmentally } \\
\text { conscious manufacturing. }\end{array}$ & Teórico-empírico & 8 \\
\hline (3) & Albert Choi, H. Kaebernick \& W. Lai & 1997 & $\begin{array}{l}\text { Manufacturing processes modeling for environmental } \\
\text { impact assessment. }\end{array}$ & Teórico-empírico & 6 \\
\hline (4) & Askiner Gungor \& Surendra Gupta & 1999 & $\begin{array}{l}\text { Issues in environmentally conscious manufacturing } \\
\text { and product recovery: a survey. }\end{array}$ & Teórico & 10 \\
\hline (5) & Joseph Sarkis & 1999 & $\begin{array}{l}\text { A methodological framework for evaluating } \\
\text { environmentally conscious manufacturing programs. }\end{array}$ & Teórico & 6 \\
\hline (6) & $\begin{array}{l}\text { David Allen, Diana Bauer, Bert } \\
\text { Bras, Timothy Gutowski, Cindy } \\
\text { Murphy, Tom Piwonka, Paul } \\
\text { Cheng, Jon Sutherland, Deborah } \\
\text { Thurston \& Egon Wolff }\end{array}$ & 2002 & $\begin{array}{l}\text { Environmentally benign manufacturing: trends in } \\
\text { Europe, Japan, and the USA. }\end{array}$ & Teórico & 7 \\
\hline (7) & $\begin{array}{l}\text { Timothy Gutowski, Cynthia } \\
\text { Murphy, David Allen, Diana Bauer, } \\
\text { Bert Bras, Thomas Piwonka, Paul } \\
\text { Cheng, Jon Sutherland, Deborah } \\
\text { Thurston \& Egon Wolff }\end{array}$ & 2005 & $\begin{array}{l}\text { Environmentally benign manufacturing: observations } \\
\text { from Japan, Europe and the United States. }\end{array}$ & Teórico & 12 \\
\hline (8) & Cathy Rusinko & 2007 & $\begin{array}{l}\text { Green manufacturing: an evaluation of } \\
\text { environmentally sustainable manufacturing practices } \\
\text { and their impact on competitive outcomes. }\end{array}$ & Teórico-empírico & 7 \\
\hline (9) & $\begin{array}{l}\text { Francesco Jovane, Hiroyuki } \\
\text { Yoshikawa, Leo Alting, Claudio } \\
\text { Boër, Engelbert Westkamper, David } \\
\text { Williams, Mitchell Tseng, Günther } \\
\text { Seliger \& Maria Paci }\end{array}$ & 2008 & $\begin{array}{l}\text { The incoming global technological and industrial } \\
\text { revolution towards competitive sustainable } \\
\text { manufacturing. }\end{array}$ & Teórico-empírico & 8 \\
\hline
\end{tabular}

Os principais autores anteriormente abordados na seção 4.3 aparecem destacados na Tabela 9 para efeito de distinção dos demais. Nota-se que a maior parte das publicações apresenta ao menos um dos autores destacados previamente. Todos os trabalhos datam do período entre 1995-2008 e, em sua maioria, envolvem autores classificados como retirantes ou one-timers.

Constata-se a partir da Tabela 9 que os estudos acerca da temática GM versam sobre assuntos teóricos (5 estudos, 55,5\%) e teórico-empíricos (4 estudos, $44,5 \%)$. Os estudos teóricos foram elaborados por meio de meta-análise, realização de revisões teóricas e proposição de frameworks. Já os estudos teóricoempíricos abordam a realização de surveys e estudos de caso em empresas.

A Tabela 10 mostra uma sintese dos principais artigos recentes (últimos 3 anos) publicados na área de GM, a partir da seleção dos artigos que citam em suas listas de referências ao menos 1 dos artigos da Tabela 9.

No total foram identificados 48 artigos recentes, apresentando LCS entre 1 e 8 . No entanto, somente os artigos listados na Tabela 10 (19 publicações - 39,6\%) citam algum dos artigos relacionados na Tabela 9. A maior parte dos autores da Tabela 10 foi classificada como entrantes ou one-timers, sendo que, novamente, os principais foram destacados em negrito. Das 19 publicações listadas, 26,3\% ocorreram em 2010,
15,8\% em 2011 e 57,9\% em 2012, sendo 52,6\% delas estudos teóricos e 47,4\% estudos teórico-empíricos.

Pela Tabela 10 constata-se que 7 publicações $(36,8 \%)$ citaram somente 1 referência da Tabela 9, 10 artigos (52,6\%) citaram 2 referências, 1 artigo $(5,3 \%)$ citou 3 referências e 1 artigo (5,3\%) citou 5 referências. As referências mais citadas nas publicações recentes foram os artigos (7), (8) e (9), normalmente utilizados como base para a fundamentação teórica das publicações recentes, visando apresentar a origem e definição do conceito GM, justificar sua importância, apresentar resultados prévios da literatura e a necessidade de se realizarem mais pesquisas na área.

Sobre os temas abordados nas publicações da Tabela 10, podem ser sistematizados os tópicos:

- Propostas de metodologias para GM;

- Propostas de metodologias para o monitoramento e avaliação do consumo de energia em processos de manufatura;

- Impactos da GM na redução de custos de processos de manufatura;

- GM e a aplicação da ACV;

- Aplicação da GM em processos de usinagem;

- Utilização da GM para a seleção de tecnologias verdes de manufatura;

- Desenvolvimento de indicadores de desempenho para GM. 
Tabela 10. Principais publicações recentes da área.

\begin{tabular}{|c|c|c|c|c|}
\hline Autoria & Ano & Temas estudados & Tipo de estudo & $\begin{array}{l}\text { Artigos da tabela } 9 \\
\text { que são citados }\end{array}$ \\
\hline Yan He \& Fei Liu & 2010 & $\begin{array}{l}\text { Methods for integrating energy consumption and } \\
\text { environmental impact considerations into the } \\
\text { production operation of machining processes. }\end{array}$ & Teórico-empírico & (3), (8) \\
\hline $\begin{array}{l}\text { Mehme Ali llgin \& Surendra } \\
\text { Gupta }\end{array}$ & 2010 & $\begin{array}{l}\text { Environmentally conscious manufacturing and } \\
\text { product recovery (ECMPRO): a review of the state of } \\
\text { theart. }\end{array}$ & Teórico & $(4),(5)$ \\
\hline $\begin{array}{l}\text { Congbo Li, Fei Liu, Xian-Chun } \\
\text { Tan \& Yanbin Du }\end{array}$ & 2010 & $\begin{array}{l}\text { A methodology for selecting a green technology } \\
\text { portfolio based on synergy. }\end{array}$ & Teórico & (4), (5), (6), (7), (8) \\
\hline $\begin{array}{l}\text { Franci Pusavec, Peter Krajnik \& } \\
\text { Janez Kopac }\end{array}$ & 2010 & $\begin{array}{l}\text { Transitioning to sustainable production - Part } 1 \text { : } \\
\text { application on machining technologies. }\end{array}$ & Teórico-empírico & (7), (9) \\
\hline Sekar Vinodh \& Gopinat Rathod & 2010 & $\begin{array}{l}\text { Integration of ECQFD and LCA for sustainable } \\
\text { product design. }\end{array}$ & Teórico-empírico & (4), (8) \\
\hline Elta Amrina \& Shari Yusof & 2011 & $\begin{array}{l}\text { Key performance indicators for sustainable } \\
\text { manufacturing evaluation in automotive companies. }\end{array}$ & Teórico & (8), (9) \\
\hline Harvey Millar \& Suzana Russell & 2011 & $\begin{array}{l}\text { The adoption of sustainable manufacturing practices } \\
\text { in the Caribbean. }\end{array}$ & Teórico-empírico & (7) \\
\hline $\begin{array}{l}\text { Perminderijt Singh \& Kuldip } \\
\text { Sangwan }\end{array}$ & 2010 & $\begin{array}{l}\text { Product and process characteristics for green } \\
\text { manufacturing: evidence from Indian SMEs. }\end{array}$ & Teórico-empírico & $(2),(4),(7)$ \\
\hline $\begin{array}{l}\text { Thomas Behrendt, André Zein \& } \\
\text { Sangkee Min }\end{array}$ & 2012 & $\begin{array}{l}\text { Development of an energy consumption monitoring } \\
\text { procedure for machine tools. }\end{array}$ & Teórico-empírico & (7) \\
\hline $\begin{array}{l}\text { Mélanie Despeisse, Peter Ball, } \\
\text { Steve Evans \& Andy Levers }\end{array}$ & 2012a & $\begin{array}{l}\text { Industrial ecology at factory level: a prototype } \\
\text { methodology. }\end{array}$ & Teórico & (1), (9) \\
\hline $\begin{array}{l}\text { Mélanie Despeisse, Peter Ball, } \\
\text { Steve Evans \& Andy Levers }\end{array}$ & $2012 b$ & $\begin{array}{l}\text { Industrial ecology at factory level - a conceptual } \\
\text { model. }\end{array}$ & Teórico & $(1),(9)$ \\
\hline $\begin{array}{l}\text { Mélanie Despeisse, Fatou Mbaye, } \\
\text { Peter Ball \& Andy Levers }\end{array}$ & $2012 c$ & $\begin{array}{l}\text { The emergence of sustainable manufacturing } \\
\text { practices. }\end{array}$ & Teórico & (1) \\
\hline $\begin{array}{l}\text { Joost Duflou, Jon Sutherland, } \\
\text { David Dornfeld, Christoph } \\
\text { Herrmann, Jack Jeswiet, Sami } \\
\text { Kara, Michael Hauschild \& Karel } \\
\text { Kellens }\end{array}$ & 2012 & $\begin{array}{l}\text { Towards energy and resource efficient manufacturing: } \\
\text { a processes and systems approach. }\end{array}$ & Teórico & (7) \\
\hline Marco Garetti \& Marco Taisch & 2012 & $\begin{array}{l}\text { Sustainable manufacturing: trends and research } \\
\text { challenges. }\end{array}$ & Teórico & (7), (9) \\
\hline $\begin{array}{l}\text { Barbara Linke, Yu-Chu Huang \& } \\
\text { David Dornfeld }\end{array}$ & 2012 & $\begin{array}{l}\text { Establishing greener products and manufacturing } \\
\text { processes. }\end{array}$ & Teórico & (6) \\
\hline $\begin{array}{l}\text { Vance Murray, Fu Zhao \& Jon } \\
\text { Sutherland }\end{array}$ & 2012 & $\begin{array}{l}\text { Life cycle analysis of grinding: a case study of non- } \\
\text { cylindrical computer numerical control grinding via a } \\
\text { unit-process life cycle inventory approach. }\end{array}$ & Teórico-empírico & (9) \\
\hline $\begin{array}{l}\text { Zhongde Shang, Shaoyan Qin, } \\
\text { Qian Liu \& Feng Liu }\end{array}$ & 2012 & $\begin{array}{l}\text { Key manufacturing technology \& equipment for } \\
\text { energy saving and emissions reduction in mechanical } \\
\text { equipment industry. }\end{array}$ & Teórico & (6), (7) \\
\hline $\begin{array}{l}\text { Sekar Vinodh, K. Jayakrishna \& } \\
\text { Dino Joy }\end{array}$ & 2012 & $\begin{array}{l}\text { Environmental impact assessment of an automotive } \\
\text { component using eco-indicator and CML } \\
\text { methodologies. }\end{array}$ & Teórico-empírico & (8) \\
\hline Sekar Vinodh \& Dino Joy & 2012 & $\begin{array}{l}\text { Structural equation modeling of sustainable } \\
\text { manufacturing practice. }\end{array}$ & Teórico-empírico & (4), (8) \\
\hline
\end{tabular}

Como tendências para pesquisas futuras, pela inexistência de um modelo consensual e abrangente que possa ser generalizado, observa-se ainda o foco no desenvolvimento de metodologias para subsidiar a $\mathrm{GM}$, visando sua integração com ferramentas gerenciais como a ACV, o desenvolvimento de indicadores de desempenho na manufatura e de critérios para a seleção de tecnologias de manufatura. Sobre os trabalhos que integram o uso da ACV, é importante ressaltar que essa técnica tem sido mais utilizada apenas para a avaliação e monitoramento do consumo de energia nos processos produtivos, não cobrindo outros aspectos ambientais relevantes, como os impactos ambientais associados às atividades de manufatura, sendo essa lacuna também uma área para o desenvolvimento de novas pesquisas.

\section{Conclusão}

Este artigo apresentou uma análise da produção científica e de tendências para futuras pesquisas em green manufacturing. Ao todo foram publicados 239 artigos no período de 1991-2013, envolvendo 589 autores de 36 países, 246 instituições e 
142 periódicos/conferências. Mais de 50\% dos artigos foram publicados nos últimos 5 anos, o que mostra a ascensão do tema.

Quanto aos países de origem das publicações, 74,3\% concentraram-se em: Estados Unidos, China, Reino Unido, Japão, Austrália, Canadá, Índia, Itália e Alemanha. Sobre as instituições envolvidas, as universidades representaram $65,4 \%$ das publicações, os institutos de pesquisa, 19,1\% e as empresas, 15,4\%. As principais instituições foram University of California (Estados Unidos), University of Chongqing (China), Wuhan University of Science and Technology (China), Technical University of Denmark (Dinamarca) e University of Cranfield (Reino Unido), as quais contribuíram com 6 a 13 publicações cada.

Sobre a análise dos periódicos/conferências mais prolíficos, os destaques foram os periódicos Journal of Cleaner Production e o International Journal of Production Research, com 19 e 10 artigos publicados, respectivamente. As publicações em conferências variaram de 1-3 artigos por evento, de modo distribuído entre as 52 conferências rastreadas, não sendo possível identificar um evento específico de destaque.

Sobre a análise de autoria das publicações, 3,4\% dos artigos foram escritos por autores entrantes, $2,1 \%$, por autores transientes, $2,1 \%$, por autores continuantes, 6,5\%, por autores retirantes e 85,7\%, por autores one-timers, o que mostra que apenas recentemente o tema estudado foi incorporado à produção científica da academia internacional. 0 autor mais produtivo em termos de número de publicações foi Feng Liu (11 artigos), seguido por David Dornfeld (10 artigos), Hua Zhang (9 artigos) e Jon Sutherland (9 artigos), sendo todos classificados como continuantes. Quanto ao número de citações GCS, os autores mais produtivos foram Suendra Gupta (368 citações), Askiner Gungor (295 citações), Joseph Sarkis (167 citações) e Richard Florida (157 citações). Todavia, os autores mais citados foram em sua maioria classificados como one-timers ou retirantes e, ainda, a maior parte das publicações ocorreu entre 1990-2007, não sendo classificadas como recentes. A partir disso inferiu-se que os autores com maior número de publicações representam os principais pesquisadores da área na atualidade e que os autores mais citados são pesquisadores que em sua maioria estão deixando de atuar na área ou que nela não são grandes atuantes.

Sobre os temas recentemente publicados destacam-se os estudos teóricos e teórico-empíricos sobre propostas de metodologias para a $\mathrm{GM}$; propostas de metodologias para o monitoramento e avaliação do consumo de energia em processos de manufatura; impactos da GM na redução de custos de processos de manufatura; GM e o uso da técnica de ACV; aplicação da GM em processos de usinagem; utilização da GM para a seleção de tecnologias verdes de manufatura; e o desenvolvimento de indicadores de performance para a GM.

Por fim, neste estudo ficou constatada a baixa participação brasileira nas pesquisas relacionadas à green manufacturing comparativamente aos líderes na área, Estados Unidos e China. 0 destaque desses países pode estar associado a fatores como a realidade fabril, produtiva e ao nível tecnológico mais sofisticado e moderno de suas indústrias em relação às do Brasil, o que fortalece a realização de pesquisas na área. Além disso, como sugestão, recomenda-se que pesquisas futuras incorporem outras bases de dados (e.g. Scopus) em estudos semelhantes.

\section{Referências}

Allen, D., Bauer, D., Bras, B., Gutowski, T., Murphy, C. Piwonka, T., Sheng, P., Sutherland, J., Thurston, D., \& Wolff, E. (2002). Environmentally benign manufacturing: trends in Europe, Japan, and the USA. Journal of Manufacturing Science and Engineering, 124, 908-920. http://dx.doi.org/10.1115/1.1505855

Amrina, E., \& Yusof, S. M. (2011). Key performance indicators for sustainable manufacturing evaluation in automotive companies. In Proceedings the IEEE International Conference on Industrial Engineering and Engineering Management, Singapore. doi:10.1109/IEEM.2011.6118084

Araújo, J. B., \& Oliveira, J. F. G. (2012). Towards a balanced scoreboard for assessing manufacturing processes sustainability. International Journal of Business Performance Management, 13, 198-221.

Associação Brasileira de Normas Técnicas - ABNT. (2009). NBR ISO 14044: Gestão ambiental - avaliação do ciclo de vida - Requisitos e orientações. Rio de Janeiro.

Behrendt, T., Zein, A., \& Min, S. (2012). Development of an energy consumption monitoring procedure for machine tools. CIRP Annals - Manufacturing Technology, 61, 4346. http://dx.doi.org/10.1016/j.cirp.2012.03.103

Bergmiller, G. G. (2006). Lean manufacturers transcendence to green manufacturing: correlating the diffusion of lean and green manufacturing systems. Florida: University of South Florida.

Choi, A. C. K., Kaebernick, H., \& Lai, W. H. (1997) Manufacturing processes modeling for environmental impact assessment. Journal of Materials Processing Technology, 70, 231-238. http://dx.doi.org/10.1016/ S0924-0136(97)00067-8

Despeisse, M., Ball, P. D., Evans, S., \& Levers, A. (2012a). Industrial ecology at factory level: a prototype methodology. Proceedings of the Institution of Mechanical Engineers, Part B: Journal of Engineering Manufacture, 226, 16481664. http://dx.doi.org/10.1177/0954405412449937

Despeisse, M., Ball, P. D., Evans, S., \& Levers, A. (2012b). Industrial ecology at factory level: a conceptual model. Journal of Cleaner Production, 31, 30-39. http://dx.doi. org/10.1016/j.jclepro.2012.02.027

Despeisse, M., Mbaye, F., Ball, P. D., \& Levers, A. (2012c). The emergence of sustainable manufacturing practices. 
Production Planning \& Control: The Management of Operations, 23, 354-376. http://dx.doi.org/10.1080/095 37287.2011.555425

Dornfeld, A. D. (2012). Green manufacturing: fundamentals and applications. Berkeley: Springer.

Duflou, J. R., Sutherland, J. W., Dornfeld, D., Hermann, C., Jeswiet, J., Kara, S., Hauschild, M., \& Kellens, K. (2012). Towards energy and resource efficient manufacturing: a processes and systems approach. CIRP Annals - Manufacturing Technology, 61, 587-609. http://dx.doi.org/10.1016/j.cirp.2012.05.002

Energy Information Administration - ElA. Annual Energy Review 2011. Retrieved from http://www.eia.gov/ totalenergy/data/annual/pdf/aer.pdf

Florida, R. (1996). Lean and green: the move to environmentally conscious manufacturing. California Management Review, 39, 80-105. http://dx.doi.org/10.2307/41165877

Garetti, M., \& Taisch, M. (2012). Sustainable manufacturing: trends and research challenges. Production Planning \& Control: The Management of Operations, 23, 83-104. http://dx.doi.org/10.1080/09537287.2011.591619

Gungor, A., \& Gupta, S. M. (1999). Issues in environmentally conscious manufacturing and product recovery: a survey. Computers \& Industrial Engineering, 36, 811-853. http:// dx.doi.org/10.1016/S0360-8352(99)00167-9

Gutowski, T., Murphy, C., Allen, D., Bauer, D., Bras, B., Piwonka, T., Sheng, P., Sutherland, J., Thurston, D., \& Wolff, E. (2005). Environmentally benign manufacturing: observations from Japan, Europe and the United States. Journal of Cleaner Production, 13, 1-17. http://dx.doi. org/10.1016/j.jclepro.2003.10.004

He, Y., \& Liu, F. (2010). Methods for integrating energy consumption and environmental impact considerations into the production operation of machining processes. Chinese Journal of Mechanical Engineering, 23, 1-8. http://dx.doi.org/10.3901/CJME.2010.04.428

Ilgin, M. A., \& Gupta, S. M. (2010). Environmentally conscious manufacturing and product recovery (ECMPRO): a review of the state of the art. Journal of Environmental Management, 91, 563-591. PMid:19853369. http:// dx.doi.org/10.1016/j.jenvman.2009.09.037

International Trade Administration - ITA. (2010). How does Commerce define Sustainable Manufacturing? Retrieved from http://www.trade.gov/competitiveness/ sustainablemanufacturing/how_doc_defines_SM.asp

Jelinski, L. W., Graedel, T. E., Laudise, R. A., McCall, D. W., \& Patel C. K. N. (1992). Industrial Ecology: concepts and approaches. Proceedings of the National Academy of Sciences of the United States of America, 89, 793-797. http://dx.doi.org/10.1073/pnas.89.3.793

Jovane, F., Yoshikawa, H., Alting, L., Boër, CR., Westkamper, E., Williams, D., Tseng, M., Seliger, G., \& Paci, AM. (2008). The incoming global technological and industrial revolution towards competitive sustainable manufacturing. CIRP Annals - Manufacturing Technology, 57, 641-659. http:// dx.doi.org/10.1016/j.cirp.2008.09.010

Kalpakjian, S. (2001). Manufacturing engineering and technology. Upper Saddle Rive: Prentice Hall.

King, A. A., \& Lenox, M. J. (2001). Lean and green? An empirical examination of the relationship between lean production and environmental performance. Production and Operations Management, 10, 244-256. http:// dx.doi.org/10.1111/j.1937-5956.2001.tb00373.x
Li, C., Liu, F., Tan, X. C., \& Du, Y. (2010). A methodology for selecting a green technology portfolio based on synergy. International Journal of Production Research, 48, 72897302. http://dx.doi.org/10.1080/00207540903382857

Linke, B., Huang, Y. C., \& Dornfeld, D. (2012). Establishing greener products and manufacturing processes. International Journal of Precision Engineering and Manufacturing 13, 1029-1036. http://dx.doi. org/10.1007/s12541-012-0134-Z

Manufuture. (2004). A vision for 2020: assuring the future of manufacturing in Europe. European Comission. (Report of the high-level group).

Merchant, M. E., Dornfeld, D., \& Wright, P. K. (2005). Manufacturing: its evolution and future. Berkeley. Retrieved from http://escholarship.org/uc/item/36d27692

Millar, H. H., \& Russell, S. N. (2011). The adoption of sustainable manufacturing practices in the Caribbean. Business Strategy and the Environment, 20, 512-526. http://dx.doi.org/10.1002/bse.707

Murray, V. R., Zhao, F., \& Sutherland, J. W. (2012). Life cycle analysis of grinding: a case study of non-cylindrical computer numerical control grinding via unit-process life cycle inventory approach. Proceedings of the Institution of Mechanical Engineers, Part B: Journal of Engineering of Manufacturing, 226, 1604-1611.

National Institute of Standards and Technology - NIST. Overview of the sustainable manufacturing. Retrieved from http://www.mel.nist.gov/msid/SSP/introduction/ manufacturing.html\#2.

Pusavec, F., Krajnik, P., \& Kopac, J. (2010). Transitioning to sustainable production. Part 1: application on machining technologies. Journal of Cleaner Production, 18, 174184. http://dx.doi.org/10.1016/j.jclepro.2009.08.010

Rusinko, C. A. (2007). Green manufacturing: an evaluation of environmentally sustainable manufacturing practices and their impact on competitive outcomes. IEEE Transactions on Engineering Management, 54, 445-454. http://dx.doi.org/10.1109/TEM.2007.900806

Sarkis, J. (1995). Manufacturing strategy and environmental consciousness. Technovation, 15, 79-97. http://dx.doi. org/10.1016/0166-4972(95)96612-W

Sarkis, J. A. (1999). Methodological framework for evaluating environmentally conscious manufacturing programs. Computers \& Industrial Engineering, 36, 793-810. http://dx.doi.org/10.1016/S0360-8352(99)00166-7

Shang, Z., Qin, S., Liu, Q., \& Liu, F. (2012). Key manufacturing technology \& equipment for energy saving and emissions reduction in mechanical equipment industry. International Journal of Precision Engineering and Manufacturing 13, 1095-1100. http://dx.doi. org/10.1007/s12541-012-0143-y

Silva, D. A. L., Delai, 1., Castro, M. A. S., \& Ometto, A. R. (2013). Quality tools applied to Cleaner Production programs: a first approach toward a new methodology. Journal of Cleaner Production, 47, 174-187. http:// dx.doi.org/10.1016/j.jclepro.2012.10.026

Silva, P. R. S., \& Amaral, F. G. (2009). An integrated methodology for environmental impacts and costs evaluation in industrial processes. Journal of Cleaner Production, 17, 1339-1350. http://dx.doi.org/10.1016/j. jclepro.2009.04.010

Silva, E. J., Ometto, A. R., Rozenfeld, H., Silva, D. A. L., Pigosso, D. C. A., \& Reis, V. R. A. (2012). Prototypal 
implementation of a remanufacturing oriented grinding machine. In D. Dornfeld \& B. S. Linke (Eds.), Proceedings of the 19th CIRP International Conference on Life Cycle Engineering (pp. 257-262). Berkeley: Springer-Verlag.

Singh, P., \& Sangwan, K. S. (2010). Product and process characteristics for green manufacturing: evidence from Indian SMEs. In Proceedings of the ASME 2010 International Manufacturing Science and Engineering Conference, New York. http://dx.doi.org/10.1115/ MSEC2010-34077

Sustainable Manufacturing Initiative. (2011). What does sustainable manufacturing mean to Australia? The CSIRO Future Manufacturing Flagship.

United Nations Environment Programme - UNEP. (2011). Towards a Green economy: pathways to sustainable development and poverty eradication. Retrieved from http://www.unep.org/greeneconomy

Vinodh, S., Jayakrshna, K., \& Joy, D. (2012). Environmental impact assessment of an automotive component using eco-indicator and CML methodologies. Clean Technologies and Environmental Policy, 14, 333-344. http://dx.doi.org/10.1007/s10098-011-0405-x

Vinodh, S., \& Joy, D. (2012). Structural equation modeling of sustainable manufacturing practices. Clean Technologies and Environmental Policy, 14, 79-84. http://dx.doi. org/10.1007/s10098-011-0379-8

Vinodh, S., \& Rathod, G. (2010). Integration of ECQFD and LCA for sustainable product design. Journal of Cleaner Production, 18, 833-842. http://dx.doi.org/10.1016/j. jclepro.2009.12.024

Walter, S. A., \& Bach, T. M. (2013). Inserção de pesquisadores entrantes na área de estratégia: análise das relações de autoria e temas estudados no período de 1997-2010. Revista Eletrônica de Administração, 19, 165-191. http:// dx.doi.org/10.1590/S1413-23112013000100007

World Commission on Environment and Development - WCED. (1987). Our common future. Oxford: Oxford University Press.

\section{Agradecimentos}

Os autores agradecem à Fundação de Amparo à Pesquisa do Estado de São Paulo - Fapesp (Processo n. 2013/06736-9) e à Coordenação de Aperfeiçoamento de Pessoal de Nível Superior - Capes (Processo n. 33002045) pelo apoio financeiro fornecido durante a realização deste estudo.

\title{
Green manufacturing: an analysis of scientific publications and trends for the future
}

\begin{abstract}
This paper presents an analysis of the international scientific publications in the green manufacturing area. We used the Web of Science database to identify 239 articles published between 1991 and 2013. The articles involved 589 authors from 36 countries, 246 institutions, and 142 journals/conferences. We found that $51.4 \%$ of the articles were published within the last five years. Among the countries involved, the most prolific were the United States and China. The most prolific journal was the Journal of Cleaner Production with 19 articles. Most of the authors, 85.7\%, were one-time authors in the field. The highest yield was 11 articles by one author, and the most cited author received 368 citations. We concluded that the green manufacturing area has increased its relevance in recent years by focusing on theoretical and empirical-theoretical studies.
\end{abstract}

Keywords

Sustainable manufacturing. Environmentally conscious manufacturing. Bibliometrics. Literature review. 